\title{
Principles for self-assembly of cyanine dyes into 2-dimensional excitonic aggregates across the visible and near infrared
}

Received 00th January 20xx, Accepted 00th January 20xx DOI: $10.1039 / \times 0 \times x 00000 x$

\author{
Arundhati P. Deshmukh, ${ }^{a}$ Austin D. Bailey, ${ }^{a}$ Leandra S. Forte, ${ }^{a}$ Xingyu Shen, ${ }^{\text {ab }}$ Niklas Geue, ${ }^{\text {ac }}$ Ellen M. \\ Sletten ${ }^{\mathrm{a}}$ and Justin R. Caram ${ }^{\mathrm{a} *}$
}

\section{Introduction}

Excitonic molecular aggregates are non-covalently assembled arrays of chromophores wherein monomeric transition dipole moments (TDMs) couple to form extended Frenkel excitons upon excitation. These aggregates exhibit emergent photophysical properties, such as extreme changes in their absorption, emission profiles and lifetimes which are tunable through aggregate topology, molecular arrangement and energetic disorder. ${ }^{1-3}$ Probing and controlling interchromophore TDM coupling is critical to engineering excitonic materials, from semiconducting polymers to artificial pigment antenna complexes. ${ }^{4,5}$ Cyanine dye aggregates are studied extensively as model excitonic systems, due to their remarkable diversity in structures (dimers, oligomers, extended sheets and nanotubes etc.), ${ }^{6,7}$ biocompatibility ${ }^{8}$ and chemical tunability. ${ }^{9,10}$ In extended J-aggregated structures, strong coupling arising from high TDMs of cyanine dyes (ranging from

a. Department of Chemistry and Biochemistry, University of California, Los Angeles, 607 Charles E. Young Dr. East, Los Angeles, California-90095, United States. b. College of Chemistry and Molecular Engineering, Peking University, Beijing 100871, P.R.China.

c. Department of Chemistry and Mineralogy, Leipzig University, Johannisallee 29, 04103 Leipzig, Germany.

† Electronic Supplementary Information (ESI) available: Characterization of aggregates (DOSY, AFM), equilibrium model derivation, supporting model results, non-negative matrix factorization, obtaining experimental Gibb's free energies, aggregation of TDBC, sugar matrix stabilization. See DOI: 10.1039/x0xx00000x<smiles>[R]C(=Cc1sc2ccc([R3])cc2[n+]1[R2])C=C1Sc2ccc([R3])cc2N1[R2]</smiles>

$$
\begin{array}{cc}
\text { Cy1: } n=0 & \mathrm{R}_{1}=\mathrm{H}, \mathrm{Me}, \mathrm{Et}, \mathrm{Ph}, \mathrm{N}(\mathrm{Ph})_{2} \\
\text { Cy3: } \mathrm{n=1} & \mathrm{R}_{2}=\mathrm{Et}, \text { Stearyl, }\left(\mathrm{CH}_{2}\right)_{\mathrm{m}_{3}} \mathrm{SO}_{3}^{-}, \\
\text {Cy5: } \mathrm{n=2} & \left(\mathrm{CH}_{2}\right)_{3} \mathrm{CO}_{2}^{-} \\
\text {Cy7: } \mathrm{n=3} & \\
& \mathrm{R}_{3}=\mathrm{H}, \mathrm{Cl}, \mathrm{OMe}
\end{array}
$$

Figure 1. General structure of benzothiazole cyanine dyes aggregated using the strategies listed in Table 1. Some examples of substituents are also listed.

$\sim 9-16 \mathrm{D})^{11}$ results in narrow linewidths, high radiative rates, and energy transfer efficiencies. ${ }^{12,13}$ Spectral tunability of cyanine dyes coupled with the large excitonic shifts afforded by long range TDM coupling make the aggregates extremely tunable across the broad spectral range from visible (400-700 $\mathrm{nm}$ ) through near and shortwave infrared (NIR: 700-1000 nm and SWIR: $1000-2000 \mathrm{~nm}$ respectively).., 9 Therefore, cyanine aggregates have extensive applications ranging from model systems for photosynthetic energy transfer, NIR/SWIR imaging, non-linear optics and plexitonics.,14-16 2-dimensional (2D) aggregates are particularly interesting due to their unique excitonic behaviors such as I-aggregation that are a direct result 
Table 1. Common aggregation strategies for benzothiazole cyanine dyes, examples of dyes from literature aggregated using the corresponding strategy. General structures of the dyes are shown in Figure 1. To simplify the vast diversity in structures, we group the dyes according to the length of cyanine bridge.

\begin{tabular}{cc} 
Aggregate preparation strategy & $\begin{array}{c}\text { Examples of common } \\
\text { benzothiazole dyes aggregated }\end{array}$ \\
\hline Direct dissolution (in water, salt & $\mathrm{Cy} 1,{ }^{17} \mathrm{Cy} 3,{ }^{18,19}$, THIATS, ${ }^{20} \mathrm{Cy} 5,^{21}$ \\
solution etc.) & $\mathrm{Cy} 7^{22}$ \\
Langmuir-Blodgett films & $\mathrm{Cy} 1^{23,24}$ \\
Adsorption on a surface & $\mathrm{Cy} 1,{ }^{25} \mathrm{Cy}^{26,27}, \mathrm{THIATS}^{28}$ \\
Thin films & $\mathrm{THIATS}^{29,30} \mathrm{Cy} 3,{ }^{31,32} \mathrm{Cy} 7^{33}$ \\
Crystallization on interface & $\mathrm{Cy} 3^{34}$ \\
DNA or polymer templating & $\mathrm{Cy} 1,{ }^{35} \mathrm{Cy} 3,{ }^{36} \mathrm{Cy} 5,{ }^{36,37} \mathrm{Cy} 7^{36}$ \\
Monomer solution injected in a & $\mathrm{Cy} 7^{7}$ (Previous work) \\
non-solvent with tunable ionic & $\mathrm{Cy3}, \mathrm{Cy} 5, \mathrm{Cy} 7$ (This work) \\
strength &
\end{tabular}

of the aggregate structure itself and thus provide a platform for chemical control of excitonic properties. 7,38

However, achieving control over aggregate structure requires chemical/physical control over the aggregation process. Controlling the aggregation of dyes presents a handle to access novel photophysics, orthogonal to traditional approach of changing chromophore structure. Understanding how these structures are stabilized and thereby controlling aggregate formation is a prerequisite to realizing their vast technological and therapeutic applications. While many studies have elucidated the excitonic features of $2 \mathrm{D}$ and tubular cyanine aggregates and related it to their supramolecular structure, ${ }^{38-40}$ little work has been done in understanding how these structures are stabilized. In Table 1, we summarize the common literature protocols used to prepare the J-aggregates with the characteristic narrow red shifted absorption for various benzothiazole cyanine dyes (Figure 1). These miscellaneous approaches involve templated growth, assisted assembly or particular solvent mixtures, demonstrating the nongeneralizable, and often non-rationalized diversity of procedures in the literature. In this manuscript, we show that a general and straightforward approach based on independent control of solvation conditions allows us to directly tune the thermodynamics of self-assembly of cyanine aggregates in solution. Thermodynamic control of self-assembly allows us to selectively stabilize an aggregate morphology under flexible conditions (such as extremely high or low dye concentrations), unlike previous methods which work under specific conditions. This makes the aggregates amenable towards a broader scope of experimental techniques, such as diffusion ordered spectroscopy (DOSY), and potential applications of aggregates in devices.

We focus on benzothiazole cyanine dyes and find that these show a simple three component equilibrium between monomers, $\mathrm{H}$-aggregates and J-aggregates (Figure 2a). Since each of these species have distinct spectroscopic signals (Figure 2a), interconversions between them are easily monitored using absorption spectroscopy. By independently controlling solvation conditions such as solvent:non-solvent ratio (methanol:water), concentration of added salt or dye concentration, we selectively stabilize a desired morphology and show tunability from one morphology to another, thereby controlling the aggregate photophysics. For the benzothiazole dyes discussed here, diffusion ordered spectroscopy (DOSY) shows that $\mathrm{H}$-aggregates are dimers while cryo-electron microscopy (cryoEM) and atomic force microscopy (AFM) reveal the J-aggregates to be extended 2D monolayer sheets. To understand the dynamic interconversions between the three species, we construct a simple three component equilibrium model that gives the equilibrium composition as spanned by a wide range of Gibb's free energies and shed light on the thermodynamic landscape of the self-assembly. We use nonnegative matrix factorization (NNMF) to show good agreement of experiments with the model, suggesting that these general principles guide cyanine aggregate self-assembly process. Finally, we apply these principles across several cyanine dyes with absorptions ranging from visible through SWIR, demonstrating that our aggregation protocol is general, and may be applied to the broad class of cyanine dyes.

\section{Experimental}

\section{Materials}

All dyes were obtained from FEW chemicals $\mathrm{GmbH}$ (catalog \# S0046, S2278, S2284, S2433 and S0837 for TDBC, Cy3-Et, Cy5$\mathrm{Ph}, \mathrm{Cy} 7-\mathrm{Ph}$ and Cy7-DPA respectively). Sodium chloride, sucrose, $D-(+)$-trehalose and methanol were obtained from Thermo Fisher Scientific. Milli-Q water (18 $\mathrm{M} \Omega$ ) was used for making all aggregate samples. All materials were used as obtained, without any further purification.

\section{Preparation and absorption of aggregate solutions}

Appropriate amounts of the dyes were weighed and dissolved in methanol to make monomer stock solutions, then diluted in methanol to make a series of monomer solutions of appropriate concentrations. Each diluted monomer solution was injected into water or aqueous $\mathrm{NaCl}$ with a specified mixing ratio. The concentration of the diluted monomer solution was decided such that the final concentration after water/ aq. $\mathrm{NaCl}$ injection is held constant. The calculations for this process were streamlined in a MATLAB code (available upon request). Glass vials used to store the samples were pre-soaked in Milli-Q water and aggregate solutions were allowed to equilibrate in dark for $24 \mathrm{hrs}$ prior to measurement. Absorption measurements were taken on Agilent Cary 60 spectrometer for visible/NIR samples. SWIR absorption spectra were taken on JASCO V-770 UV/Vis/NIR spectrometer. Starna Cells quartz cuvettes of path lengths $0.01 \mathrm{~mm}, 0.2 \mathrm{~mm}$ or $1 \mathrm{~mm}$ were used depending on the dye concentration.

\section{Cryo-electron microscopy (cryoEM)}

Samples were prepared on mesh 200 lacey formvar/carbon copper grids obtained from Ted Pella Inc. The grids were plasma-cleaned under a $\mathrm{H}_{2} / \mathrm{O}_{2}$ gas flow using a Solarus Gatan Plasma cleaner for hydrophilization. Vitrobot Mark IV was used 
a

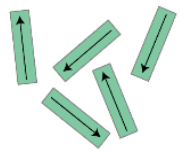

Monomers

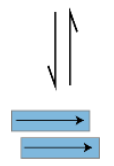

$\mathrm{H}$-aggregates

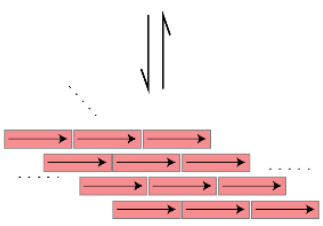

J-aggregates
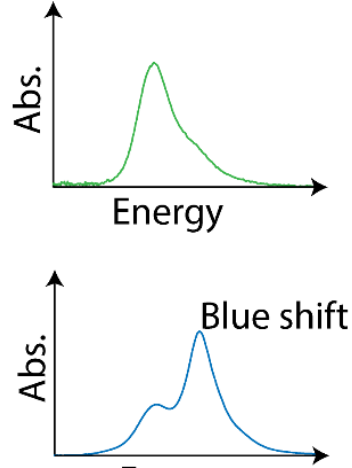

Energy

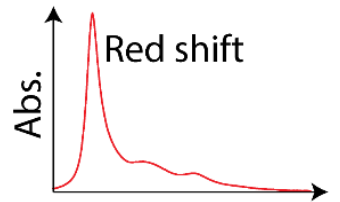

Energy b

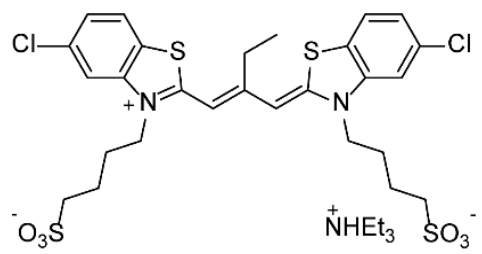

Cy3-Et

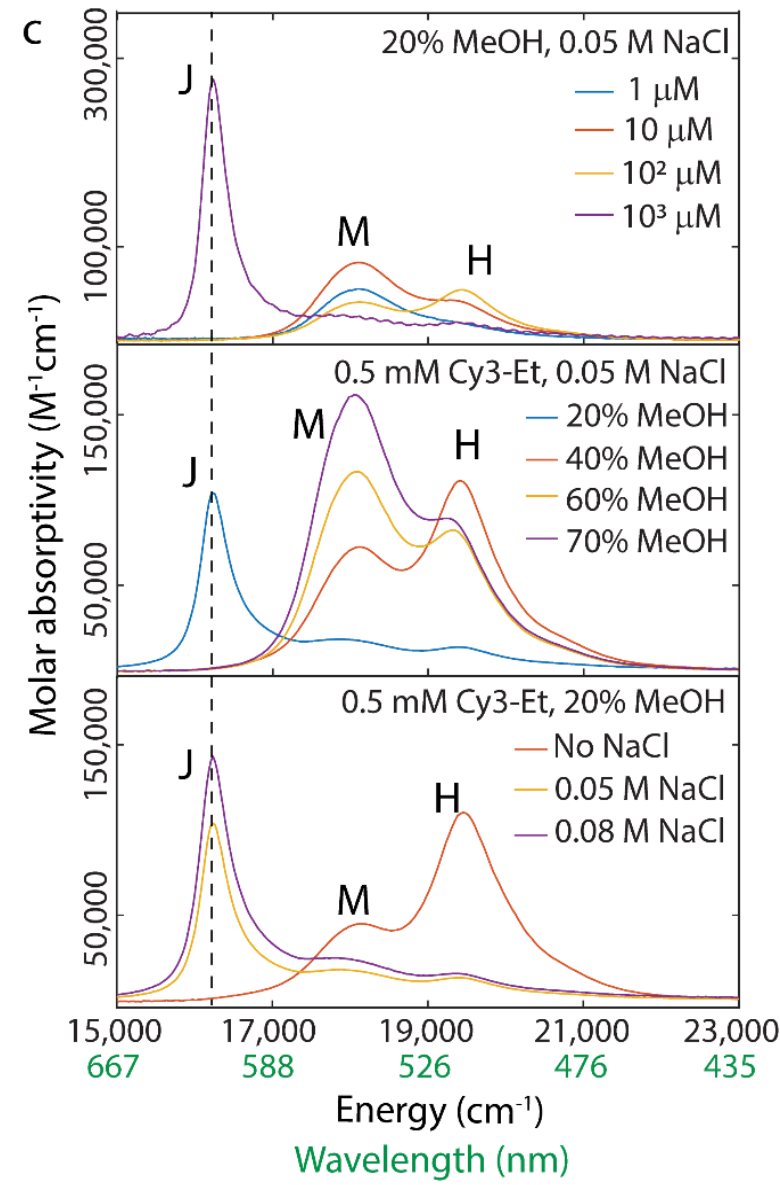

Figure 2. a. Schematic showing the general transformation of monomers to $\mathrm{H}$-aggregates (dimers) to J-aggregates (extended monolayer sheets) and characteristic lineshapes of absorption spectra of the corresponding species in solution; b. Structure of the dye Cy3-Et; c. Selected absorption spectra taken 24 hours after sample preparation where a predissolved monomer solution in methanol was injected into aqueous solution with or without $\mathrm{NaCl}$ while varying (top) dye concentration, (middle) v/v methanol:water ratio, and (bottom) salt concentration. In each case, rest of the two factors were kept constant.

for plunge-freezing the samples. $5 \mu \mathrm{L}$ of the aggregate solution was dropped onto the grid, and excess solution was removed by blotting for $3.0 \mathrm{~s}$ with standard blotting paper from Ted Pella and immediately dropped into liquid ethane. The frozen grids were stored in liquid nitrogen. Grids were loaded on to a liquid nitrogen cooled Gatan 626 cryo-transfer sample holder, then inserted into the microscope. CryoEM images were recorded on a FEI TF20 electron microscope equipped with a field-emission gun at $200 \mathrm{keV}$ and CCD camera.

\section{Atomic force microscopy (AFM)}

Films for AFM were prepared according to a previously reported drop-flow technique with some modifications. ${ }^{6}$ Aggregate solutions (2-3 drops) were dropped on a freshly cleaved mica substrate $(1 \mathrm{~cm}$ diameter) kept at a small incline. Mica substrates were obtained from Thermo Fisher Scientific. The solutions were allowed to slowly air dry on the bench while covered with a box to shield from room light and air currents. AFM measurements were done on Veeco Bioscope II AFM with tapping mode Al coated AFM tips obtained from NanoAndMore USA Corp. with $42 \mathrm{~N} / \mathrm{m}$ force constant and a resonant frequency of $\sim 320 \mathrm{kHz}$.

\section{Diffusion ordered spectroscopy (DOSY) NMR}

NMR spectra were taken at room temperature on a Bruker Avance $500 \mathrm{MHz}$ spectrometer with a $5 \mathrm{~mm}$ broadband probe and processed using TopSpin 4.07. After finding sufficient values for gradient pulse length ( $\mathrm{p} 30, \delta)$ and diffusion time (d20, $\Delta$ ) to yield spectra with 95 and $5 \%$ of original signal, 32 spectra were taken on a linear ramp and processed through TopSpin to get diffusion data.

\section{Sugar matrix stabilization}

Prepared solutions of 2D aggregates were mixed with a saturated sugar solution made by dissolving a 50:50 sucrose/trehalose $(\mathrm{w} / \mathrm{w})$ in distilled water. $100 \mu \mathrm{L}$ aggregate solution was taken in a vial pre-soaked in Milli-Q water. To this, $100 \mu \mathrm{L}$ of the sugar solution was added drop-wise and gently mixed. The sugar-aggregate mixture was dropped onto a 0.2 , 0.1 or $0.01 \mathrm{~mm}$ quartz cuvette from Starna Cells Inc. and kept under vacuum in dark for $24 \mathrm{~h}$.

\section{Results and discussion}


a
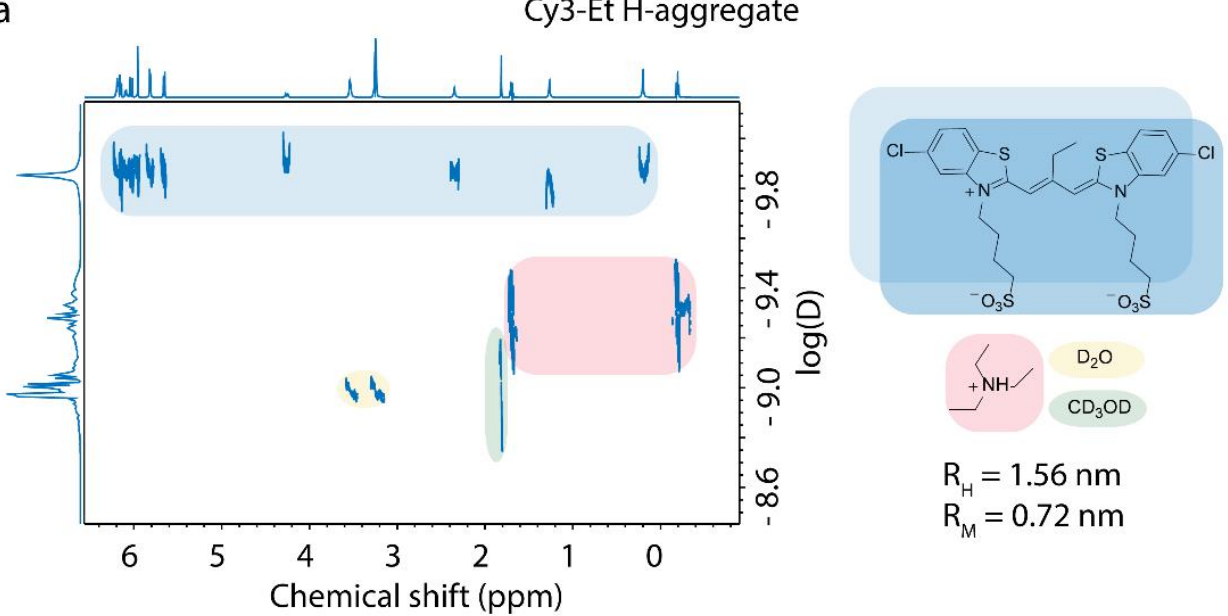

b

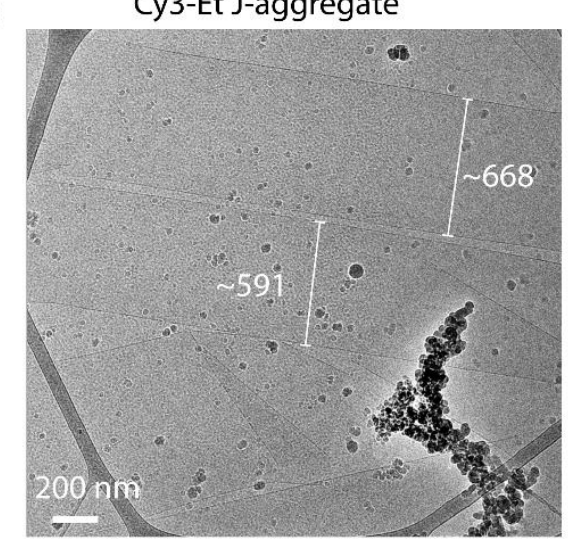

c

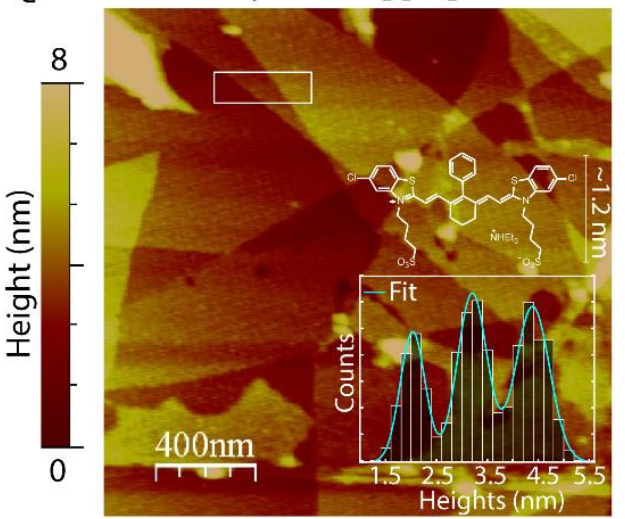

Figure 3. a. $1 \mathrm{H}$ diffusion ordered spectroscopy (DOSY) of Cy3-Et H-aggregates at $1 \mathrm{mM}$ concentration prepared in $90 \% \mathrm{D} 2 \mathrm{O}$ and $10 \% \mathrm{MeOD}$. Comparing hydrodynamic radius with monomer suggests dimer morphology; b. Cryo-electron microscopy image of vitrified Cy3-Et J-aggregate depicting sheet-like morphology. Numbers indicate sheet widths in nanometers; c. Atomic force microscopy image of Cy7-Ph J-aggregates with a histogram of the heights (bottom inset) enclosed in the white rectangle. The histogram is fit to a sum of three gaussians (cyan line) with $\mathrm{R}^{2}=0.9695$. Height of a single layer obtained from the histogram matches the height of a single molecule (top inset) indicating monolayer sheets.

\section{Aggregation studies}

We use the simplified nomenclature scheme Cyn- $R$ for all the benzothiazole cyanine dyes in this study where $n$ denotes the number of carbons on the cyanine bridge and $R$ denotes the substitution on central position. Throughout this paper, we will use commercially obtained dye 3,3'-bis(4-sulfobutyl)-5, $5^{\prime}$ dichloro-9-ethylthiacarbocyanine, ammonium salt or Cy3-Et (Figure 2b) as a model system to explore the thermodynamics of self-assembly. Cy3-Et is structurally related to the commonly studied THIATS chromophore, differing only in the number of carbons ( 3 vs 4 ) in the sulfoalkyl chain. ${ }^{29}$ Several strategies have been used to aggregate THIATS and other benzothiazole cyanine dyes, summarized in the Table 1 , including direct dissolution in water, ${ }^{22}$ adsorption of silver halide surface, ${ }^{26}$ and spin coating. ${ }^{29}$ Here, we stabilize Cy3-Et aggregates under a variety of conditions by independent control of different solvation conditions. First, we make a monomer solution of the dye in methanol and disperse it into an aqueous solution (with or without $\mathrm{NaCl}$ ), a more generalized modification of the wellknown 'alcoholic route'. ${ }^{6}$
We use these three parameters to access the aggregation phase space, and plot the resulting absorption spectra in Figure 2c. We vary (i) final dye concentration (top panel); (ii) solvent to non-solvent ratio (middle panel); and (iii) ionic strength by changing added salt concentration (bottom panel). In each case, the other two factors are kept constant demonstrating orthogonal tunable conditions and allowing us to span a large range of experimental conditions. Unsurprisingly, we find that higher dye concentrations favor $\mathrm{H}$-aggregation followed by $\mathrm{J}$ aggregation. In addition, high \% $\mathrm{MeOH}(\sim 70 \%)$ and low dye concentrations always favor monomeric state. As we decrease the $\% \mathrm{MeOH}$ or increase the salt concentration, we observe gradual transformation of monomers to $\mathrm{H}$-aggregate and finally to J-aggregate. Our results are consistent with the hypothesis that ionic strength induced dielectric screening and hydrophobic interactions (lower \% $\mathrm{MeOH}$ ) stabilize extended aggregates. $^{41}$ Moreover, this far simpler and easily generalizable approach yields the similar narrow red shifted absorption lineshapes for J-aggregates, as seen in previous methods (listed in Table 1), indicating that we are able to access the similar J-aggregate microstructures that lead to this characteristic lineshape with higher degree of control. 


\section{Characterization of $\mathrm{H}$ - and J-aggregate structures}

Outside of changing the concentration of dye, the use of mutually distinct solvation conditions (e.g. salt, solvent to nonsolvent ratio) to stabilize the desired $\mathrm{H}$ - or J-aggregate state allows us to develop preparations that are amenable to characterization tools requiring high concentrations of a single species. For example, smaller aggregate species like dimers and oligomers are too small for characterization tools such as dynamic light scattering, cryoEM or AFM. However, multidimensional NMR methods such as diffusion ordered spectroscopy (DOSY) typically require very high concentrations where fully aggregated species tend to be more dominant than smaller aggregates. Using the methods outlined above, we were able to perform DOSY on $\mathrm{H}$-aggregates, stabilizing the $\mathrm{H}$ aggregate at a concentration of $1 \mathrm{mM}$ by retaining a low volume fraction of deuterated methanol (10:90 MeOD: $\mathrm{D}_{2} \mathrm{O}$, no $\mathrm{NaCl}$ ). As shown in Figure $3 a$, all the peaks corresponding to the aggregate emerge at a single diffusion constant $1.32 \times 10^{-10} \mathrm{~m}^{2} / \mathrm{s}$ while faster diffusing species such as counter ions and solvent show up at higher diffusion constants. A comparison with the DOSY spectrum of the monomer is shown in Supporting Information (SI) Figure S1. The monomer also shows a unique diffusion constant for all dye protons at a higher value of $4.96 \mathrm{x}$ $10^{-10} \mathrm{~m}^{2} / \mathrm{s}$. The hydrodynamic radii obtained using StokesEinstein relationship, given in Figure $3 a$, show that the $\mathrm{H}$ aggregate is about twice the size of the monomer (see SI Table $\mathrm{S} 1$ for details). We thus conclude that the $\mathrm{H}$-aggregate is a dimer consistent with previous concentration dependent absorption studies with similar dyes. ${ }^{18}$

For the redshifted J-aggregates, we perform CryoEM and AFM, tools for characterizing larger mesoscale structures (Figures $3 \mathrm{~b}-\mathrm{c}$ ). CryoEM demonstrates extended rectilinear sheets with average dimensions of $500-1000 \mathrm{~nm}$ by several microns. We then use AFM analysis to probe the height of these 2D sheets and analyze the layer structure. As Cy3-Et J-aggregate solutions require added salt, complicating height analysis, Cy7$\mathrm{Ph}$ J-aggregates (prepared without salt at $20 \% \mathrm{MeOH}, 0.2 \mathrm{mM}$ dye as described in our prior work) were analyzed. ${ }^{7}$ AFM images of Cy7-Ph (Figure 3c and SI Figure S2) are fit to the difference in heights between adjacent layers. ${ }^{6}$ We plot a histogram of the heights of a selected region (Figure $3 \mathrm{c}$ bottom inset) and fit it to a sum of 3 Gaussians,

$$
\mathrm{y}=\sum_{i=1}^{3} \mathrm{a}_{\mathrm{i}} \cdot \mathrm{e}^{-\left(\frac{x-b_{i}}{c_{i}}\right)^{2}}
$$

where $a_{i}$, and $b_{i}$ respectively are the amplitudes, center positions of the Gaussians and and $c_{i}$ are related to width. The height of a single sheet was calculated from the difference in the center positions of adjacent peaks $\left(b_{i+1}-b_{i}\right)$, and is $1.18 \pm$ $0.01 \mathrm{~nm}$, which matches the height of a Cy7-Ph molecule 1.2 $\mathrm{nm}$ (Figure 3c, top inset). Thus, we conclude that the sheets are monolayers with the molecular plane perpendicular to the plane of the sheets. Since we do not observe significant differences in spectral lineshapes or electron microscope images for different dyes studied here (detailed discussion later in Figure 6), we believe that this aggregate morphology applies to all J-aggregates studied.

\section{Three component equilibrium model}

We construct a thermodynamic model that describes the energetic landscape governing self-assembly of dimerized $\mathrm{H}$ aggregates and sheet-like J-aggregates. Similar approaches have been used to describe other aggregation processes, including isodesmic and cooperative self-assembly, however most studies focus one-dimensional aggregate structures. ${ }^{41,42}$ Based on the observations in Figure 2c, we consider two general equilibria as follows.

$$
\begin{aligned}
& n_{1} M \rightleftharpoons H \\
& n_{2} H \rightleftharpoons J
\end{aligned}
$$

$M, H$, and $J$ denote the monomer, $\mathrm{H}$-aggregate and $\mathrm{J}$ aggregate species respectively. $n_{1}$ denotes the number of monomers in an $\mathrm{H}$-aggregate and $n_{1} \cdot n_{2}$ is the total number of monomers in the J-aggregate. We use the subscripts 1 and 2 for the equilibrium constants $\left(K_{e q}\right)$ and standard Gibb's free energies $\left(\Delta G^{\circ}\right)$ of equilibria (1) and (2) respectively, which can be related as $\Delta G^{\circ}=-R T \ln \left(K_{e q}\right)$ where $R$ and $T$ are the universal gas constant and temperature respectively. The respective equilibrium constants can be written as

$$
K_{1}=\frac{C_{H}}{C_{M}^{n_{1}}} ; K_{2}=\frac{C_{J}}{C_{H}^{n_{2}}}
$$

The total concentration, $C_{t o t}$ is defined as

$$
C_{t o t}=C_{M}+n_{1} \cdot C_{H}+n_{1} \cdot n_{2} \cdot C_{J}
$$

where $C_{M}, C_{H}$ and $C_{J}$ denote the concentrations of monomer, $\mathrm{H}$ aggregate and J-aggregate respectively. Upon substitution and rearrangement (see SI Section 2 for a complete derivation), we arrive at the characteristic polynomial of $C_{M}$.

$$
\begin{gathered}
n_{1} \cdot n_{2} \cdot K_{2} \cdot K_{1}^{n_{2}} \cdot C_{M}^{n_{1} \cdot n_{2}}+n_{1} \cdot K_{1} \cdot C_{M}^{n_{1}}+C_{M}-C_{t o t} \\
=0
\end{gathered}
$$

This high order polynomial will always have exactly one real positive root (proof in SI Section 2), which allows us to use standard root finding methods to find $C_{M}$ for a given set of parameters. Thereafter, mole fractions of each species $\left(\chi_{M}, \chi_{H}\right.$ and $\left.\chi_{J}\right)$ can be calculated for any combination of $\Delta G^{\circ}$ using equations (3-5). Thus, we can uniquely define the equilibrium composition of the solution for any combination of standard Gibb's free energies.

In Figure 4a-c, we map the ternary equilibrium composition as we change the standard Gibb's free energies of $\mathrm{H}$-aggregated dimerization on the $\mathrm{x}$-axis and extended 2D J-aggregate formation on the $y$-axis. We set $n_{1}=2$ based on our DOSY results (Figure $3 \mathrm{a}$ ) and $n_{2}=10$ representing total aggregation number of 20 in extended J-aggregates. While this number greatly underestimates the number of aggregated monomers, it captures the sharp onset of J-aggregation as a function of concentration. As a noteworthy point, each point on these maps represents a different equilibrium condition for a fixed concentration. Given a set of experimental $\Delta G^{o ' s}$ for any system, our model predicts the equilibrium composition at any 

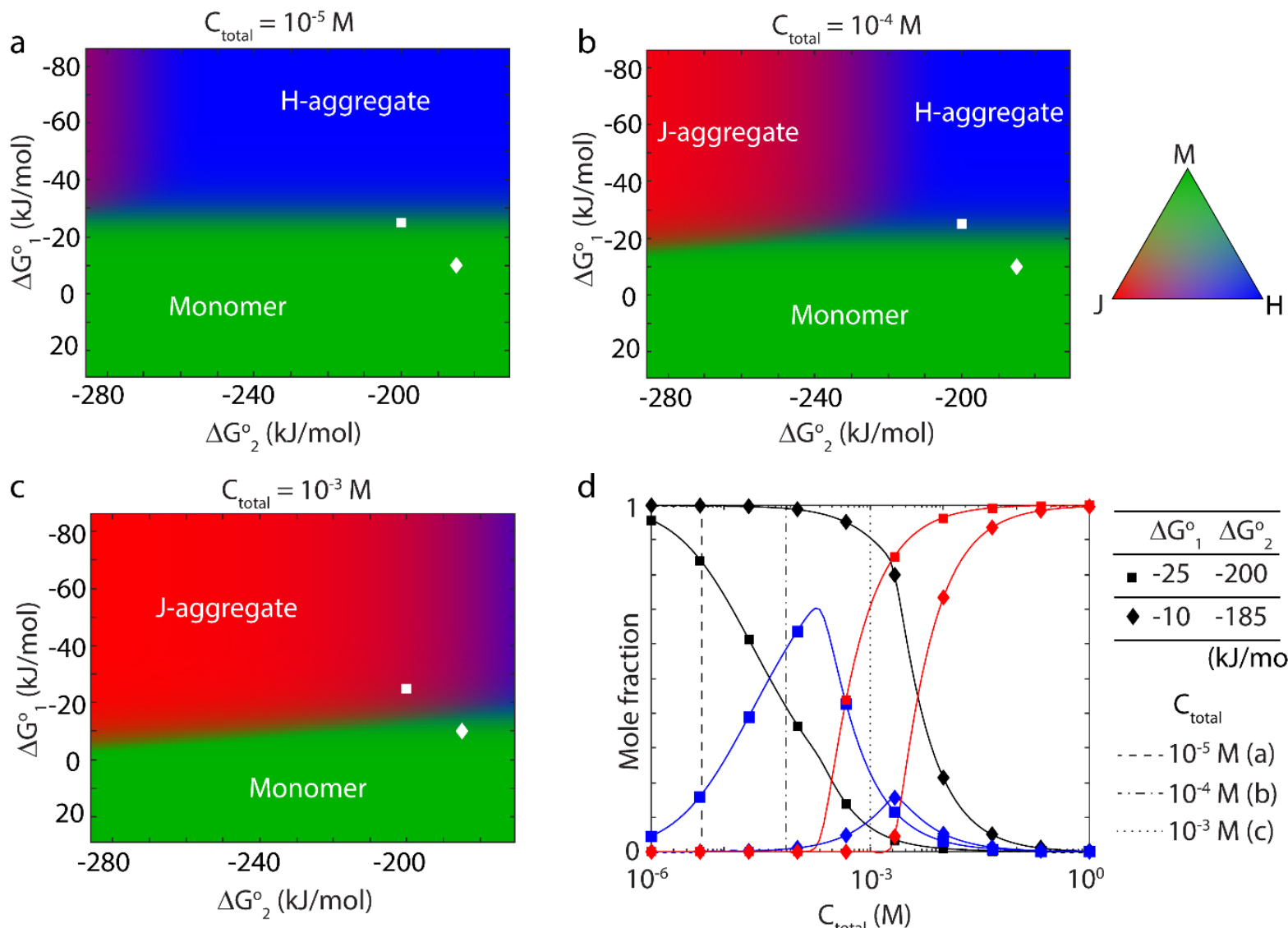

d

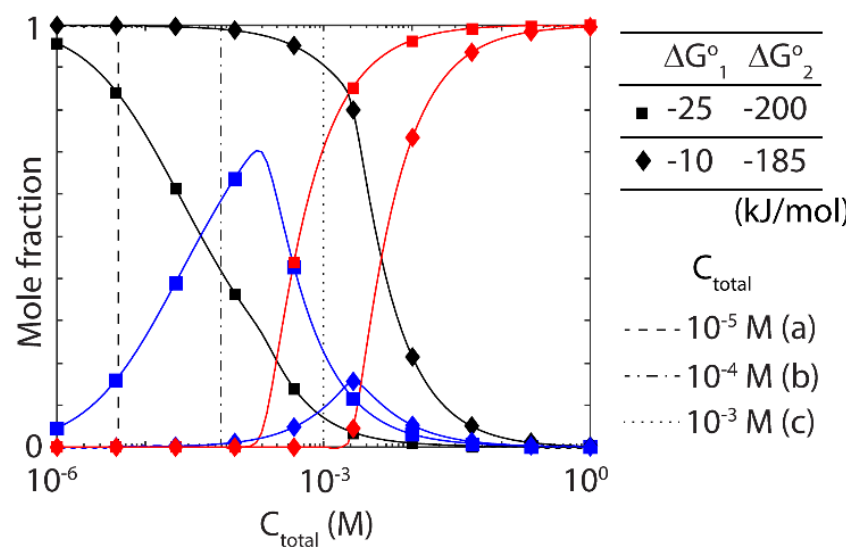

Figure 4. Prediction of equilibrium composition using the three component model at any concentration. a-c. Maps showing the variation of equilibrium composition as a function of standard Gibb's free energies of monomer to $\mathrm{H}$-aggregate equilibrium $\left(\Delta G_{1}^{\circ}\right)$ and $\mathrm{H}$-aggregate to J-aggregate $\left(\Delta G_{2}^{\circ}\right)$ for different total dye concentrations $\left(C_{\text {total }}\right), n_{1}=2, n_{2}=$ 10, a. $C_{\text {total }}=10^{-5} \mathrm{M} ;$ b. $C_{\text {total }}=10^{-4} \mathrm{M}$; and c. $C_{\text {total }}=10^{-3} \mathrm{M}$. The colour of each pixel denotes the composition shown in the ternary plot on the right with RGB value corresponding to mole fractions of J-aggregate, monomer and $\mathrm{H}$-aggregate respectively; $\mathrm{d}$. Evolution of mole fractions of monomer (black), $\mathrm{H}$-aggregate (blue) and J-aggregate (red) with total dye concentration at two points on the $\Delta G^{\circ}$ maps denoted by square $\left(\Delta G_{1}^{\circ}=-25, \Delta G_{2}^{\circ}=-200 \mathrm{~kJ} / \mathrm{mol}\right)$ and diamond $\left(\Delta G_{1}^{\circ}=-10, \Delta G_{2}^{\circ}=-185 \mathrm{~kJ} / \mathrm{mol}\right)$. Three vertical lines correspond to the $C_{\text {total }}$ values in parts a-c.

concentration as shown in Figures 4a-c. We span this aggregation space by changing the solvation conditions such as $\% \mathrm{MeOH}$ or salt concentration which directly affect $\Delta G_{1}^{\circ}$ and $\Delta G_{2}^{\circ}$, demonstrating thermodynamic control. Another way to span this map is by adding substituents to the chromophore that might affect one or both of these values. Direct transition from $\mathrm{H}$-aggregate to J-aggregate involves a change in $\Delta G_{2}^{\circ}$ (corresponding to extended aggregate formation). This suggests that increasing the ionic strength at low $\% \mathrm{MeOH}$ (Figure 2c) mostly changes $\Delta G_{2}^{\circ}$. At highly negative $\Delta G_{2}^{\circ}$, we observe a direct transition from monomer to J-aggregate upon reducing $\Delta G_{1}^{\circ}$. However, in our experiments, we observe that this equilibrium goes through a dimer $\mathrm{H}$-aggregate possibly due the extremely large size of the actual aggregates which would require a higher limiting threshold concentration to form the $2 \mathrm{D}$ aggregate structure.

We pick two fixed points on the maps in Figures $4 a-c$ corresponding to $\Delta \mathrm{G}_{1}^{\circ}=-25, \Delta \mathrm{G}_{2}^{\circ}=-200 \mathrm{~kJ} / \mathrm{mol}$ (square), and $\Delta \mathrm{G}_{1}^{\circ}=-10, \Delta \mathrm{G}_{2}^{\circ}=-185 \mathrm{~kJ} / \mathrm{mol}$ (diamond). In Figure $4 \mathrm{~d}$, we show how the mole fractions of each component evolve with total dye concentration for these combinations of $\Delta G^{o \text { 's }}$. Three vertical lines correspond to the concentrations in Figures 4a-c. In all the cases, J-aggregates require a certain threshold concentration where we observe a sharp increase or 'turn-on' in the J-aggregate mole fraction. This non-sigmoidal nature of Jaggregate mole fraction curves is indicative of cooperative mechanism which plays an important role in the formation of large aggregates. ${ }^{41}$ In SI Figure S3, we show mole fractions as a function of $n_{2}$ which depicts the delayed and sharper turn-on in J-aggregation consistent with larger aggregate structures. This threshold can be lowered by increasing the ionic strength of the solution as seen from Figure $2 c$ (bottom panel) where we observe a shift form $\mathrm{H}$ - to J-aggregate. We hypothesize that increasing the ionic strength directly affects $\Delta G_{2}^{\circ}$ due to additional charge screening, which is more important in stabilizing larger charged aggregates. This is also visible from SI Figure S4, where $\Delta G_{1}^{o}$ does not affect the J-aggregate onset as much as $\Delta G_{2}^{o}$ concurrent with a sharp decrease in monomer and $\mathrm{H}$-aggregate.

This sharp turn-on in J-aggregate as one increases concentration is a clear indication of cooperative self-assembly akin to a phase transition or reaching a critical micelle concentration. ${ }^{43,44}$ We attribute the cooperativity to the large number of non-covalent interactions that drive the formation of 


\section{a Non-negative matrix factorization}
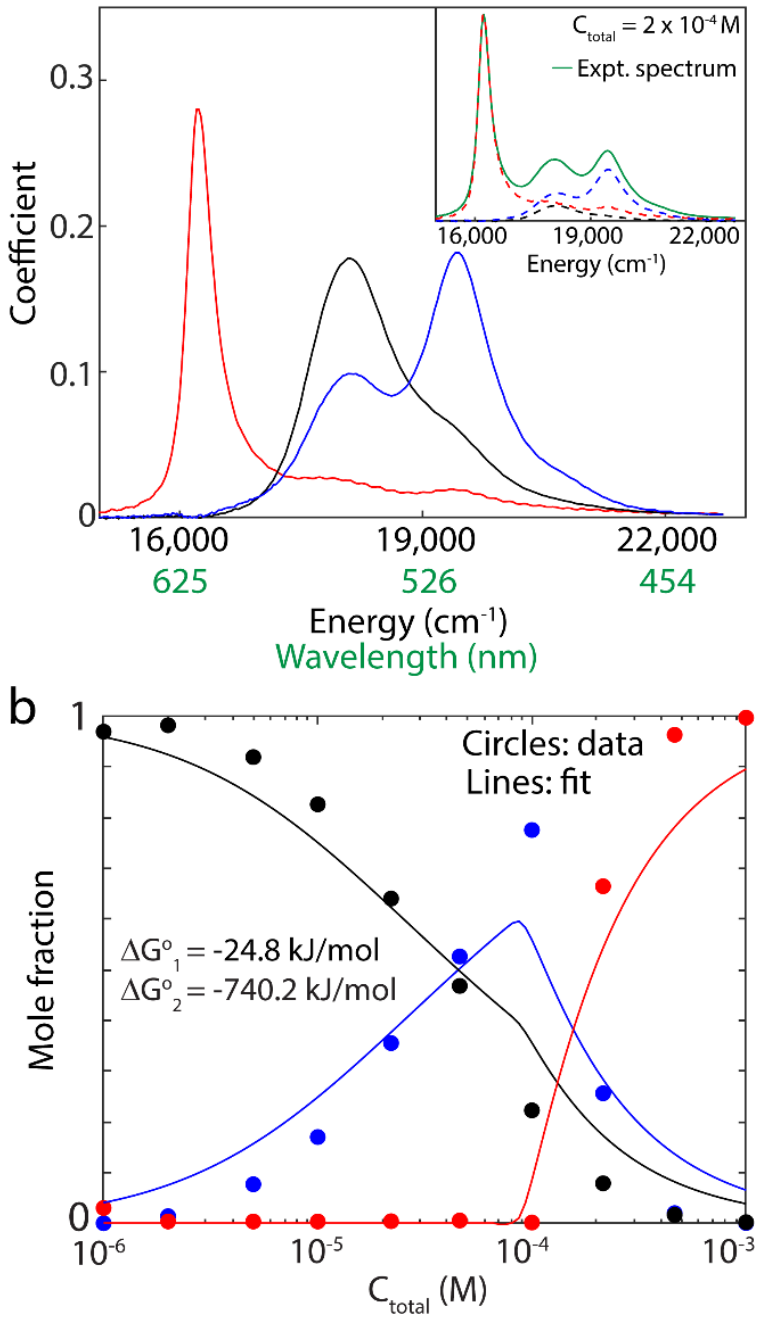

Figure 5. a. Basis vectors obtained from non-negative matrix factorization (NNMF) of experimental concentration dependence data (shown in SI Figure S5); (Inset) Example of an experimental spectrum (green) with the decomposition into three NNMF vectors (dashed lines) corresponding to monomer (black), H-aggregate (blue), and J-aggregate (red); b. Experimental mole fractions obtained from NNMF (circles) and the fitted mole fraction curves from the three component model (lines). For $\mathrm{n}_{1}=2, \mathrm{n}_{2}=30$, parameters obtained by least squares curve fitting are $\Delta G_{1}^{\circ}=-24.8 \mathrm{~kJ} / \mathrm{mol}, \Delta G_{2}^{\circ}=-740.2 \mathrm{~kJ} / \mathrm{mol}$.

2D J-aggregate sheets. In two dimensions the number of potential aggregate contacts increases linearly with the aggregate size. ${ }^{43}$ Therefore, one can achieve cooperative assembly without invoking a change in chemical potential for every additional monomer, unlike more widely-studied 1D systems. Furthermore, our results suggest that many systems can be aggregated into 2D brick-layer sheets simply by stabilizing $\Delta G_{2}^{\circ}$ relative to $\Delta G_{1}^{\circ}$, for example, through control of solvent to non-solvent ratio or by adding salt. Critically, in most cases once a threshold concentration is reached monomers or smaller aggregates simultaneously transform into the large Jaggregate as seen from the sharp decline in the monomer and $\mathrm{H}$-aggregate mole fractions (Figure 4d).

In order to show quantitative agreement between our model and experiment, we perform a concentration dependence of Cy3-Et at $20 \% \mathrm{MeOH}$ and $0.05 \mathrm{M} \mathrm{NaCl}$ (shown in
SI Figure S5). Since there are three overlapping spectra (corresponding to each species) at equilibrium, one cannot apply Beer's law to determine mole fractions of each species. Instead, we compare the experiments to our model by decomposing our observed spectra into $\mathrm{J}, \mathrm{H}$, and $\mathrm{M}$ spectra via non-negative matrix factorization (NNMF). NNMF can be used deconvolve the spectra of pure components from inseparable mixtures (see SI Section 4 for details). ${ }^{45}$ By factorizing a 2D array of mixed spectra into a pre-chosen number of basis vectors ( 3 in this case) the corresponding weights can be used to find mole fraction of chromophores in each aggregate form. We perform NNMF on a set of concentration dependent spectra (SI Figure S5) and obtain three basis vectors shown in Figure 5a, which are consistent with experimental spectra of the monomer, and published spectra for dimer $\mathrm{H}$-aggregates and $\mathrm{J}$-aggregate species (Figure 2a). ${ }^{18}$ As an example, we show the deconvolution of an experimental spectrum into individual monomer, $\mathrm{H}$ - and J-aggregate components in the inset of Figure $5 a$. We plot the mole fractions obtained from NNMF for this concentration series in Figure $5 b$ (circles), capturing the sharp increase in the J-aggregate at $10^{-4} \mathrm{M}$.

Finally, we fit the mole fraction data for all three components simultaneously to arrive at Gibb's Free energies. First, to decrease the fitting space, we derive a simple analytical expression for $\Delta G_{1}^{o}$ using the monomer to $\mathrm{H}$-aggregate crossing point, under the assumption of an infinitely large aggregate. At the monomer to $\mathrm{H}$-aggregate crossing point, we apply the condition $\chi_{M}=\chi_{H}$ to the characteristic polynomial from equation (6) and using limit $n_{2} \rightarrow \infty$, we obtain an analytical equation, $\Delta G_{1}^{o}=R T \ln \left(C_{t o t}^{\prime}\right)$, where $C^{\prime}{ }_{\text {tot }}$ is the dye concentration where the mole fractions of monomer and $\mathrm{H}$ aggregate are equal (see SI Section for 5 derivation). Interpolating the experimental mole fractions obtained from NNMF in Figure $5 \mathrm{~b}$, we obtain $C^{\prime}{ }_{\text {tot }}=40.8 \mu \mathrm{M}$ which results in the analytically obtained value of $\Delta G_{1}^{\circ}=-25.0 \mathrm{~kJ} / \mathrm{mol}$. We then fit our three component model to this data (details in SI section 5), finding good agreement for $n_{1}=2$ and $n_{2}=30$ (greater values of $n_{2}$ are numerically unstable) and optimize the values of $\Delta \mathrm{G}_{1}^{\circ}=-24.8 \mathrm{~kJ} / \mathrm{mol}, \Delta \mathrm{G}_{2}^{\circ}=-740.2 \mathrm{~kJ} / \mathrm{mol}$ using leastsquares curve fitting (lines in Figure $5 \mathrm{~b}$ ). The highly negative $\Delta G_{2}^{\circ}$, indicating the strong favorability of extended $2 \mathrm{D}$ aggregate formation, is ascribed to the increased number of interactions in $2 \mathrm{D}$ aggregates. ${ }^{44}$ Given the actual size of the aggregates is much larger, the formation of extended 2D aggregates is probably extremely cooperative due to the increased number of pair-wise interactions between the monomers with increasing aggregate size, unlike 1D aggregates where it does not increase with size. ${ }^{44}$

\section{Generality of self-assembly principles}

In Figure 6, we demonstrate that principles for selfassembly used here are truly generalizable and are also extendable to other families of cyanine dyes such as benzimidazole cyanines. Figure 6 shows a list of cyanine dyes that form 2D sheet-like aggregates with tunable absorption from visible through SWIR. In all these cases, the aggregation behavior followed similar trends where lower \% $\mathrm{MeOH}(20$ - 
Dye

a

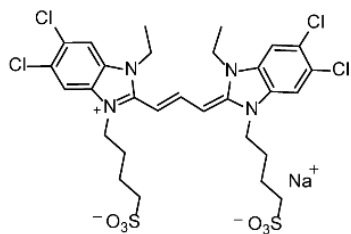

TDBC

b

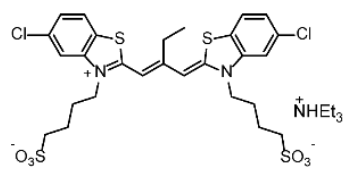

Cy3-Et

C

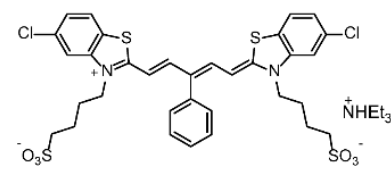

Cy5-Ph

d

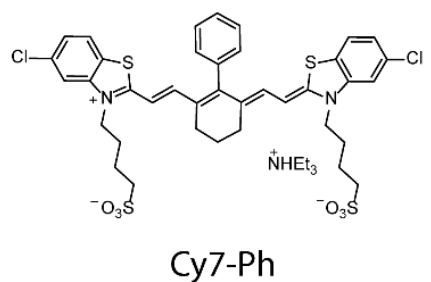

e

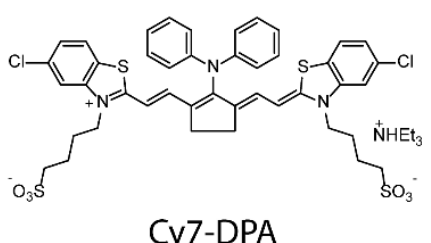

\section{CryoEM of J-aggregate}
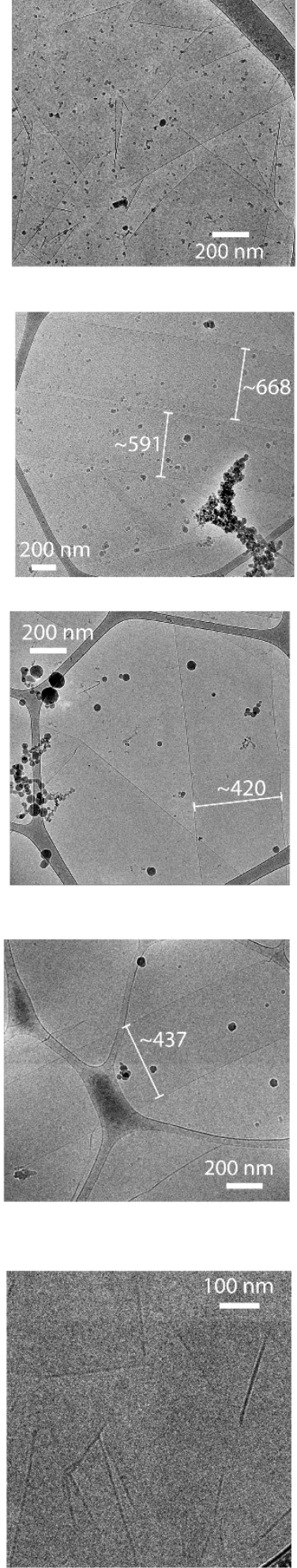

Absorption spectra
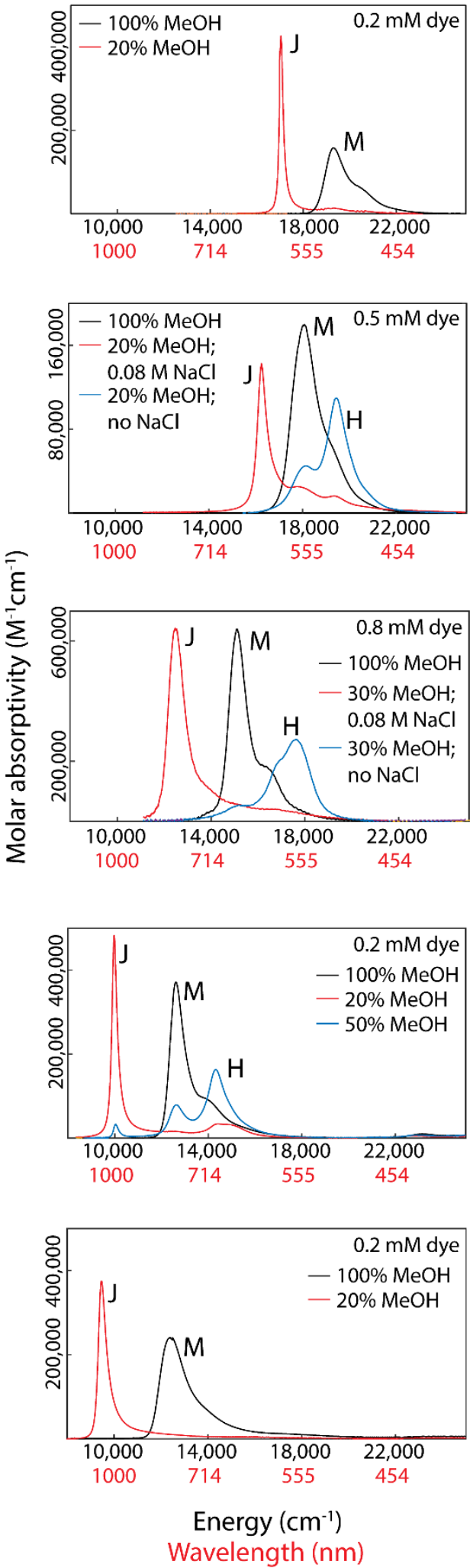

Figure 6. Applying the general principles from this work to several dyes with absorption tunability from visible through SWIR: (left) dye structures; (center) cryo-electron microscopy image of the 2D sheet aggregates and (right) absorption spectra of monomer (black), H-aggregate (blue) and red shifted 2D aggregate (red). Numbers on cryoEM images indicate sheet widths in nanometers.

$30 \%)$ gave the extended J-aggregated sheets, the optimal salt With the exceptions of TDBC and Cy7-DPA, we also observe and dye concentrations differ slightly among individual dyes.

similar $\mathrm{H}$-aggregate peak at $50 \% \mathrm{MeOH}$. Particularly, larger dye 
molecules like Cy7-DPA and Cy7-Ph aggregate readily without any need for salt where as Cy3-Et and Cy5-Ph need slightly higher dye concentrations with added salt, suggesting that higher surface areas lead to stronger van der Waals interactions. Going beyond benzothiazole cyanine dyes, we are also able to apply these principles on different classes of cyanine dyes. A particular example of the well-known benzimidazole cyanine dye, 1,1'-diethyl-3,3'-bis(4-sulfobutyl)$5,5^{\prime}, 6,6^{\prime}$-tetrachlorobenzimidazolocarbocyanine or TDBC is also shown in Figure 6a. TDBC is also known to form sheet aggregates that have garnered interest in excitonic energy transfer and polaritonics. ${ }^{14,46}$ We show the principles outlined above can also be used to tune the self-assembly of TDBC into sheet like J-aggregates (see SI Figure S6 for details). Unlike rest of the dyes, TDBC and Cy7-DPA do not go through an $\mathrm{H}$ aggregate state likely due the ethyl groups (in case of TDBC) and the bulky diphenylamine group (in case of Cy7-DPA) that might sterically hinder cofacial stacking. Furthermore, we show that all the 2D aggregates from Figure 6 can be stabilized in a sugar matrix (SI Figure S7) which is known to protect the aggregates against photo and air damage, ${ }^{12}$ also facilitating cryogenic and high excitation flux spectroscopies on the excitonic aggregates.

\section{Conclusions}

Our aggregation strategy based on independently controlling the solvent to non-solvent ratio, salt or dye concentration provides a general and tunable avenue for selectively stabilizing an aggregate morphology for the broad class of cyanine dyes. Our approach avails a broad range of aggregation conditions to stabilize a desired morphology, which in turn, controls the photophysical behavior. In addition, tunability over a vast aggregation space enables new structural characterization techniques such as DOSY and broadens the application space. For example, aggregating at lower concentrations through lower non-solvent ratio can be used to control the optical density of aggregate antennas in thin films.

We explain the conserved aggregation trends on the basis of a simple three component equilibrium model and gain insights into the thermodynamics of the self-assembly process. Our results show that ionic strength mainly effects $\Delta G_{2}^{\circ}$, enabling direct transition from dimer $\mathrm{H}$-aggregates to extended J-aggregates at the same dye concentration and solvent composition, likely by stabilizing larger charged structures. Solvent to non-solvent ratio, on the other hand, likely affects both $\Delta G_{1}^{\circ}$ and $\Delta G_{2}^{\circ}$ as it can induce both $\mathrm{H}$ - and J-aggregation depending on the available dye concentration. NNMF untangles the constituent spectra and shows we can achieve excellent agreement between model and experiment. Finally, we show that solvation control can be extended to several sheet forming benzothiazole as well as benzimidazole cyanine dyes thus enabling a tunable library with absorptions ranging from visible through SWIR.

The kinetics and thermodynamics of aggregation of chromophores is a topic of extensive research, mostly focused on 1-dimensional $\pi$-stacking structures of porphyrins and perylene bisimides. ${ }^{41,42,47}$ However, extended 2-dimensional aggregates have unique features not reproduced in linear aggregates. The number of pair-wise interactions between an aggregate and a monomer is proportional to the aggregate size in the 2D case, whereas it is a constant for linear aggregate. In analogy to bilayer formation in surfactants, one observes a near phase transition above a set concentration of dye in 2D aggregates. $^{44}$ In linear aggregates with pairwise interactions, phase transitions are rigorously forbidden. ${ }^{44}$ In addition, despite the rich excitonic properties and diverse applications of cyanine dye aggregates, application of the thermodynamic selfassembly principles to the broad class of cyanine dyes aggregates is unprecedented. Overall, 2D aggregation provides a unique platform for even more stable structures at lower concentrations while retaining the excitonic features of Jaggregates and the broad class of cyanine dyes is an excellent avenue for this. The simple and broadly applicable principles for aggregation presented here provide the first step toward realizing many potential applications

\section{Conflicts of interest}

No conflict of interest.

\section{ORCiD}

Arundhati P. Deshmukh: 0000-0002-7901-8814

Niklas Geue: 0000-0002-5216-8353

Ellen M. Sletten: 0000-0002-0049-7278

Justin R. Caram: 0000-0001-5126-3829

\section{Acknowledgements}

This work was supported by NSF CHE grant no. 190524 and Faculty Research Grant (UCLA Academic Senate). APD thanks UCLA Chemistry and Biochemistry Excellence in Research Fellowship and SG Fellowship for financial support. Authors thank Materials Characterization Laboratory, UCLA for instrumentation and Dr. Timothy L. Atallah, UCLA for constructive comments. APD thanks Sudhanshu Srivastava, UCSB for discussions on statistical methods. XS thanks CSST (Cross-disciplinary Scholars in Science and Technology) Scholarship. Authors acknowledge the use of instruments at the Electron Imaging Center for NanoMachines supported by NIH (1S10RR23057 to ZHZ) and CNSI at UCLA.

\section{References}

1 M. Kasha, Radiat. Res., 1963, 20, 55-71.

2 A. S. Davydov, A. S. D. ydov, M. Kasha and M. Oppenheimer, Theory of Molecular Excitons, McGraw-Hill, 1962.

3 H. Fidder, J. Knoester and D. A. Wiersma, J. Chem. Phys., 1991, 95, 7880-7890.

4 T. Brixner, R. Hildner, J. Köhler, C. Lambert and F. Würthner, Adv. Energy Mater., 2017, 7, 1700236.

5 H. Yamagata and F. C. Spano, J. Chem. Phys., 2012, 136, 184901. 
D. M. Eisele, J. Knoester, S. Kirstein, J. P. Rabe and D. A. Vanden Bout, Nat. Nanotechnol., 2009, 4, 658-663. A. P. Deshmukh, D. Koppel, C. Chuang, D. M. Cadena, J. Cao and J. R. Caram, J. Phys. Chem. C, 2019, 123, 18702-18710. C. Sun, B. Li, M. Zhao, S. Wang, Z. Lei, L. Lu, H. Zhang, L. Feng, C. Dou, D. Yin, H. Xu, Y. Cheng and F. Zhang, J. Am. Chem. Soc., 2019, 141, 19221-19225.

J. L. Bricks, Y. L. Slominskii, I. D. Panas and A. P. Demchenko, Methods Appl. Fluoresc., 2017, 6, 012001.

A. Pawlik, A. Ouart, S. Kirstein, H.-W. Abraham and S. Daehne, European J. Org. Chem., 2003, 2003, 3065-3080. A. Petrenko and M. Stein, J. Phys. Chem. A, 2019, 123, 9321-9327.

J. R. Caram, S. Doria, D. M. Eisele, F. S. Freyria, T. S. Sinclair, P. Rebentrost, S. Lloyd and M. G. Bawendi, Nano Lett., 2016, 16, 6808-6815.

S. Doria, T. S. Sinclair, N. D. Klein, D. I. G. Bennett, C. Chuang, F. S. Freyria, C. P. Steiner, P. Foggi, K. A. Nelson, J. Cao, A. Aspuru-Guzik, S. Lloyd, J. R. Caram and M. G. Bawendi, ACS Nano, 2018, 12, 4556-4564.

X. Zhong, T. Chervy, S. Wang, J. George, A. Thomas, J. A. Hutchison, E. Devaux, C. Genet and T. W. Ebbesen, Angew. Chemie Int. Ed., 2016, 55, 6202-6206.

5 P. A. Bouit, G. Wetzel, G. Berginc, B. Loiseaux, L. Toupet, P. Feneyrou, Y. Bretonnière, K. Kamada, O. Maury and C. Andraud, Chem. Mater., 2007, 19, 5325-5335.

D. M. Eisele, D. H. Arias, X. Fu, E. A. Bloemsma, C. P. Steiner, R. A. Jensen, P. Rebentrost, H. Eisele, A. Tokmakoff, S. Lloyd, K. A. Nelson, D. Nicastro, J. Knoester and M. G. Bawendi, Proc. Natl. Acad. Sci., 2014, 111, E3367-E3375.

H. Bücher and H. Kuhn, Chem. Phys. Lett., 1970, 6, 183 185.

D. Takahashi, H. Oda, T. Izumi and R. Hirohashi, Dye. Pigment., 2005, 66, 1-6.

X. Chen, Y. H. Chen, J. Qin, D. Zhao, B. Ding, R. J. Blaikie and M. Qiu, Nano Lett., 2017, 17, 3246-3251.

I. G. Scheblykin, O. P. Varnavsky, W. Verbouwe, S. De Backer, M. Van Der Auweraer and A. G. Vitukhnovsky, Chem. Phys. Lett., 1998, 282, 250-256.

H. V Berlepsch and C. Böttcher, Phys. Chem. Chem. Phys., 2018, 20, 18969-18977.

H. V Berlepsch and C. Böttcher, J. Photochem. Photobiol. A Chem., 2010, 214, 16-21.

A. Yamaguchi, N. Kotnetani and Y. Yonezawa, J. Phys.

Chem. B, 2005, 109, 1408-1414.

P. Debnath, S. Chakraborty, S. Deb, J. Nath, B. Dey, D. Bhattacharjee, H. Soda, M. Tominaga, Y. Suzuki, J.

Kawamata and S. A. Hussain, Appl. Clay Sci., 2017, 147, 105-116.

B. Laban, V. Vodnik, M. Dramićanin, M. Novaković, N. Bibić S. P. Sovilj and V. M. Vasić, J. Phys. Chem. C, 2014, 118, 23393-23401.

K. Kemnitz, K. Yoshihara, T. Suzumoto, T. Tani, M. Lindrum, J. Moll and S. Daehne, Primary photophysical processes in J-aggregates of spectral sensitisers, 1993, vol. 105.

F. Zhang, K. Sautter, A. M. Larsen, D. A. Findley, R. C. Davis,

28

H. Samha and M. R. Linford, Langmuir, 2010, 26, 1464814654.

J. W. Oh, S. Kumazaki, I. V. Rubtsov, T. Suzumoto, T. Tani and K. Yoshihara, Chem. Phys. Lett., 2002, 352, 357-362. D. Hasegawa, K. Nakata, E. Tokunaga, K. Okamura, J. Du and T. Kobayashi, J. Phys. Chem. A, 2013, 117, 1144111448.

Y. Tanaka, H. Yoshikawa and H. Masuhara, in Optical Trapping and Optical Micromanipulation III, eds. K. Dholakia and G. C. Spalding, SPIE, 2006, vol. 6326, p. 63262J.

K. Chevrier, J. M. Benoit, C. Symonds, J. Paparone, J. Laverdant and J. Bellessa, ACS Photonics, 2018, 5, 80-84. V. V. Prokhorov, S. I. Pozin, D. A. Lypenko, O. M. Perelygina, E. I. Mal'tsev and A. V. Vannikov, Macroheterocycles, 2012, 5, 371-376.

C. Wang and E. A. Weiss, Nano Lett., 2017, 17, 5666-5671. S. Kirstein, H. Möhwald and M. Shimomura, Chem. Phys. Lett., 1989, 154, 303-308.

O. K. Kim, J. Je, G. Jernigan, L. Buckley and D. Whitten, J. Am. Chem. Soc., 2006, 128, 510-516.

R. A. Garoff, E. A. Litzinger, R. E. Connor, I. Fishman and B. A. Armitage, Langmuir, 2002, 18, 6330-6337.

K. C. Hannah and B. A. Armitage, Acc. Chem. Res., 2004, 37, 845-853.

C. Chuang, D. I. G. Bennett, J. R. Caram, A. Aspuru-Guzik, M. G. Bawendi and J. Cao, Chem, 2019, 5, 3135-3150. C. Didraga, A. Pugžlys, P. R. Hania, H. Von Berlepsch, K. Duppen and J. Knoester, J. Phys. Chem. B, 2004, 108, 14976-14985.

D. M. Eisele, C. W. Cone, E. A. Bloemsma, S. M. Vlaming, C. G. F. van der Kwaak, R. J. Silbey, M. G. Bawendi, J. Knoester, J. P. Rabe and D. A. Vanden Bout, Nat. Chem., 2012, 4, 655-662.

Z. Chen, A. Lohr, C. R. Saha-Möller and F. Würthner, Chem. Soc. Rev., 2009, 38, 564-584.

M. M. J. Smulders, M. M. L. Nieuwenhuizen, T. F. A. De Greef, P. Van Der Schoot, A. P. H. J. Schenning and E. W. Meijer, Chem. - A Eur. J., 2010, 16, 362-367.

A. Ben-Shaul and W. M. Gelbart, Springer, New York, NY, 1994, pp. 1-104.

J. N. Israelachvili, Intermolecular and Surface Forces, Elsevier, 3rd edn., 2011.

A. S. Montcuquet, L. Hervé, F. Navarro, J. M. Dinten and J. I. Mars, IEEE Trans. Biomed. Eng., 2011, 58, 2554-2565.

Q. Hu, D. Jin, J. Xiao, S. H. Nam, X. Liu, Y. Liu, X. Zhang and N. X. Fang, Proc. Natl. Acad. Sci. U. S. A., 2017, 114, 1001710022.

T. Fukui, S. Kawai, S. Fujinuma, Y. Matsushita, T. Yasuda, T. Sakurai, S. Seki, M. Takeuchi and K. Sugiyasu, Nat. Chem., 2017, 9, 493-499. 